\title{
Editorial
}

\section{Immunology of chronic mucocutaneous candidiasis}

Patients with chronic mucocutaneous candidiasis (CMC) are susceptible to debilitating, persistent, and refractory infections of the skin, nails, and mucous membranes with yeasts of the genus candida, most often by the species Candida albicans. The underlying defect is thought to be an impairment of immune defences but the nature of the defect(s) has not been defined. ${ }^{1}$ Candida albicans is an opportunistic yeast inhabiting, as a commensal, the mucous membranes of $>80 \%$ of adults, becoming invasive only if the existing balance is disrupted. Various non-immunological factors are known to predispose to candida overgrowth and thrush (diabetes, obesity, systemic antibiotics, systemic and local steroid treatment, dentures, and occlusion-for example, nappies and tight clothing, etc); however, these infections are characteristically resolved as soon as the predisposing factors are removed and should not be confused with a persistent inability to clear candida, as is seen in CMC. ${ }^{2}$ Patients with CMC do not constitute a homogenous group and numerous classifications have been proposed, mostly based on the presence or absence of endocrinopathy, juvenile or mature onset, and familial or sporadic occurrence. ${ }^{3}$ Associated findings include alopecia, vitiligo, malabsorption disorders, and infections with encapsulated microorganisms. ${ }^{4}$ Patients with CMC and endocrinopathy are now frequently referred to as having the APECED (autoimmune polyendocrinopathy candidiasis ectodermal dysplasia) syndrome, ${ }^{5}$ previously known as APS-1 (autoimmune polyendocrinopathy syndrome type 1). In these patients, a genetic defect has recently been identified showing (various) mutations in the AIRE (autoimmune regulator) gene, which probably encodes a DNA transcription factor. The link between the identified gene defect and the inability to clear candida remains unknown. No gene defect has as yet been identified in patients with CMC without endocrinopathy, although there have been occasional reports of such patients where multiple family members through several generations were affected, again suggesting an underlying gene defect. ${ }^{6}$ Research in this area is currently in progress in our and other laboratories. *

\section{Immunity to candida}

Protective immunity to candida is complex and includes both the innate response and adaptive cellular and humoral immune responses. ${ }^{1}$ Data suggest that different mechanisms operate in protection from systemic as opposed to mucocutaneous disease, and indeed disseminated disease is rarely seen in patients with CMC and vice versa. Protection from systemic disease and internal organ spread is mediated primarily by innate immunity, particularly by neutrophils and complement. ${ }^{7}$ Neutrophil and macrophage function is dependent on antibody and complement opsonisation, as well as pattern recognition receptors such as the mannose and Toll-like receptors. ${ }^{89}$

^Because affected families with CMC but without endocrinopathy are extremely rare, it would be much appreciated if any physicians/immunologists seeing or being aware of such patients would contact the authors.
Intracellular killing is mediated primarily through oxygen dependent pathways (superoxide anion, myeloperoxidase and reactive nitrogen (NO) production), although oxygen independent pathways (lysozymes) have also been implicated. ${ }^{10}$ Most at risk from systemic candidiasis are patients who develop granulocytopenia, such as patients on immunosuppressive or cytotoxic treatment for malignancies and other diseases, or patients with inborn phagocytic defects (such as chronic granulomatous disease and hyper-IgE syndrome). ${ }^{11}$ Interestingly, although complement greatly enhances candida phagocytosis, patients with complement component deficiencies including C3 and C5-9 are not more susceptible. ${ }^{11}$ Several groups have argued that antibodies to candida are crucial in protection from widespread disease, particularly an antibody specific for a $47 \mathrm{kDa}$ candida protein. ${ }^{12}$ However, this is difficult to reconcile with the fact that patients with humoral (antibody) immune deficiencies are not particularly prone to infections with fungi, including candida ${ }^{11}$ Indeed, patients with CMC have repeatedly been shown to have high amounts of candida specific antibody, ${ }^{13}$ but are still unable to clear the infection. Interestingly, although the role of $\mathrm{T}$ cells is paramount in regulating the humoral and, to a certain degree, the innate immune responses described above, $\mathrm{T}$ cell mediated effector mechanisms seem to be crucially important in protection against mucocutaneous rather than systemic spread. Earlier studies showed that candida itself, in particular candida mannan, exerts immunosuppressive properties that result in depressed T cell function. ${ }^{14}$ Although this might contribute to the immune depression seen in patients with CMC, this is unlikely to play a major role because it is well documented that patients with CMC regularly relapse in spite of candida eradication by vigorous antifungal treatment. $^{2}$

\section{Immunity in chronic mucocutaneous candidiasis}

INNATE IMMUNITY

Research into the immune defect(s) in patients with CMC over the years mostly demonstrated intact innate immunity to candida, including complement function as well as neutrophil phagocytosis and intracellular killing. ${ }^{1}$ However, the data on macrophage function are controversial, suggesting subtle impairment of macrophage activation and intracellular killing. ${ }^{15}$ Most importantly, the regulatory (cytokine producing) role of the macrophage in CMC remains largely unknown.

\section{HUMORAL IMMUNITY}

Antibody responses in general and specifically to candida have repeatedly been shown to be intact. ${ }^{13}$ Our own data showed very high titres of IgG1 and IgA candida specific antibodies in all patients. However, recent data suggest that a proportion of patients with CMC may have impaired antibody responses to encapsulated bacteria such as Haemophilus influenzae B and Streptococcus pneumoniae, with or without accompanying IgG2 subclass deficiency. ${ }^{4}$ 
T CELL MEDIATED IMMUNITY AND CYTOKINES

Protection from mucocutaneous candidiasis has repeatedly been shown to be dependent on cellular immunity and, although the defect remains elusive, it is clear that patients lacking $T$ cells (for example, severe combined immune deficiencies, Di George syndrome), and in particular $\mathrm{CD}^{+} \mathrm{T}$ cells (patients with AIDS), will suffer from this form of candidiasis. ${ }^{11}$ In recently identified patients with inborn deficiencies of the receptors for interferon $\gamma$ (IFN- $\gamma$ ) and interleukin 12 (IL-12) it was shown that, apart from being susceptible to atypical mycobacteria, some also had persistent candidiasis. ${ }^{16}$ Although our own studies have excluded IFN- $\gamma$ receptor/IL-12 receptor deficiency as a possible cause of susceptibility to chronic candidiasis in patients with CMC (vide infra), it is tempting to speculate that in analogy a rather general immune defect may be underlying the seemingly specific susceptibility to candida. In a series of well designed experiments in animal models, protection against candidiasis was shown to be dependent on a type 1 pattern of cytokine production. ${ }^{17}$ These studies demonstrated that IFN- $\gamma$ and IL-12 were required for survival and clearance of infection, ${ }^{18}$ whereas neutralising IL-4 in susceptible strains improved survival. ${ }^{19}$ However, an animal model of spontaneous CMC is unavailable and the relevance of these findings for CMC in humans remains to be confirmed. In patients with CMC the abnormalities identified were found to affect the cellular arm of the immune response. A spectrum of defects was reported, ranging from no detectable abnormalities through selective absence of responses to candida antigens to a generalised absence of responses to ubiquitous delayed type hypersensitivity antigens, both in vivo and in vitro (delayed type hypersensitivity skin tests, proliferation assays, macrophage/leucocyte migration inhibition assays). In general, standard lymphocyte subset analysis (CD3/CD4/ CD8/CD19) yielded normal results. ${ }^{13}$ Taken together, these data suggested that the defect in CMC was at the level of cell mediated immunity, most likely involving $\mathrm{T}$ cells, possibly the $\mathrm{CD}^{+}$subset, and affected soluble mediators. However, the defect remained undefined. Recently, our preliminary data demonstrated that patients with CMC had an altered pattern of cytokine production in response to candida antigens, with low or absent IL-2 production, increased IL-6 production, and very high titres of IgG1 and IgA candida specific antibodies, consistent with a low type 1 and high type 2 cytokine production pattern. ${ }^{13}{ }^{20}$ Our current, more comprehensive studies on a larger number of patients with CMC, looking at a range of inflammatory as well as type 1 and type 2 cytokines, demonstrated increased production of inflammatory cytokines in response to candida antigens (tumour necrosis $\alpha(\mathrm{TNF}-\alpha)$ and IL-6) as well as a significant increase in IL-10 production in all patients. This was parallelled by decreased values of IL-12, IFN- $\gamma$, and (to a lesser degree) IL-2. Interestingly, concentrations of IL-4 and IL- 5 were low and comparable with controls. These alterations in cytokine production were more pronounced upon stimulation with purified carbohydrate candida fractions and mannan than with protein fractions (our data, unpublished, 2000).

Taken together, our data demonstrate for the first time that patients with CMC have a defect that presents as altered cytokine production in response to candida antigens. We speculate that these patients may be "over-reacting" to candida, possibly to carbohydrate moieties such as mannan, glucan, or chitin, by overproducing inflammatory cytokines that in turn trigger a feedback overproduction of IL-10. High IL-10 values could then lead to downregulation of the protective type 1 response, which could explain the low IL-12 values that were found in our patients. Interestingly, our data did not demonstrate increased type 2 cytokine production, suggesting that it is the insufficient production of type 1 rather than overproduction of type 2 cytokines that undermines the efficient clearance of candida in these patients. It is of note that all of our patients expressed normal amounts of receptors for IFN- $\gamma$ and IL-12 (T Lammas, personal communication, 2000). At this point, it is impossible to say whether the defect primarily lies with the $\mathrm{T}$ cells as type 1 cytokine producers or whether the fault lies further "upstream", involving accessory cells (macrophages?), which fail to produce adequate amounts of type 1 inducing cytokines, such as IL-12. Although the mechanisms are unclear, our data suggest that patients with CMC may also have a problem in the recruitment/maturation of candida specific $\mathrm{CD}^{+} / \mathrm{CD}^{+}$cells, which fail to switch from $\mathrm{CD} 45 \mathrm{RA}^{+}$to $\mathrm{CD} 45 \mathrm{RO}^{+}$after culture with candida antigens (our data, unpublished, 1999): this could result in altered cytokine producing properties of the cells involved. Cytokine production by macrophages and/or responsiveness to cytokine stimulation in response to candida is currently also under study. In conclusion, the immune defect underlying susceptibility to candida infections in patients with CMC affects cell mediated responses through altered cytokine production, resulting in the production of inadequate concentrations of type 1 cytokines. The question of what skews cytokine responses remains unanswered, although the likely candidate is the macrophage, either through its antigen presenting or cytokine producing function. This hypothesis is currently under investigation in our laboratory. These findings have bearing not only on understanding CMC itself but also on understanding the mechanisms involved in protective immunity to fungi in general, which are still poorly understood.

This work was supported by registered charity Action Research. Thanks to J H Robinson and M Abinun for helpful suggestions and C Coulton for expert technical assistance.

D LILIC

I GRAVENOR

Department of Microbiology and Immunology, The Medical School, University of Newcastle, Framlington Place, Newcastle NE2 4HH, UK desa.lilic@ncl.ac.uk

1 Bodey GP, ed. Candidiasis. Pathogenesis, diagnosis and treatment, 2nd ed. New York: Raven Press, 1993:167-84.

2 Anonymous. Guidelines of care for superficial mycotic infections of the skin mucocutaneous candidiasis. I Am Acad Dermatol 1996;34:110-15.

Kirkpatrick CH. Chronic mucocutaneous candidiasis. f Am Acad Dermatol 1994;31:S14-17.

4 Herrod HG. Chronic mucocutaneous candidiasis in childhood and complications of non-candida infection: a report of the pediatric immunodeficiency collaborative study group. F Pediatr 1990;116:377-32.

5 Peterson P, Nagamine K, Scott $\mathrm{H}$, et al. APECED: a monogenic autoimmune disease providing new clues to self-tolerance. Immunol Today 1998;19:384-6.

6 Sams WM, Snyderman R, Jegasothy BV, et al. Chronic mucocutaneous candidiasis. Immunologic studies of three generations in a single family. $\mathrm{Am} \mathcal{F}$ Med 1979;67:948-58.

7 Romani L, Menacacci A, Cenci E, et al. Neutrophils and the adaptive immune response to Candida albicans. Res Immunol 1996;147:512-18.

8 Stahl PD, Ezecowitz RAB. The mannose receptor is a pattern recognition receptor involved in host defence. Curr Opin Immunol 1998;10:50-5.

9 O'Neill LAJ, Dinarello CA. The IL-1 receptor/toll-like receptor superfamily: crucial receptors for inflammation and host defence. Immunol Today 2000;21:206-9.

10 Vasquez-Torres A, Balish E. Macrophages in resistance to candidiasis. Microbiol Mol Biol Rev 1997;61:170-92.

11 Primary immunodeficiency diseases. Report of an IUIS scientific group. Clin Exp Immunol 1999;118(suppl 1): 17 .

12 Matthews R, Smith D, Midgley J, et al. Candida and AIDS: evidence for protective antibody. Lancet 1988;2:263-6.

13 Lilic D, Calvert JE, Cant AJ, et al. Chronic mucocutaneous candidiasis: II Class and subclass of specific antibody responses in vivo and in vitro. Clin Exp Immunol 1996;105:213-19.

14 Durandy A, Fischer A, Le Deist F, et al. Mannan-specific and mannan-induced T-cell suppressive activity in patients with chronic mucocutaneous candidiasis. F Clin Immunol 1983;71,1602-13.

15 Ashman RB, Papadimitriou JM. Production and function of cytokines in natural and acquired immunity to Candida albicans infection. Microbiol Rev 1995;59:646-72.

16 Ottenhoff HM, Kumararatne D, Casanova JL. Novel human immunodeficiencies reveal the essential role of type-1 cytokines in immunity to intracellular bacteria. Immunol Today 1998;19:491-4.

17 Romani L. The T cell response against fungal infections. Curr Opin Microbiol 1999;2:363-7.

18 Cenci E, Mencacci A, Del Sero G, et al. IFN $\gamma$ is require for IL-12 responsiveness in mice with Candida albicans infection. F Immunol 1998;161:3543-50. 
19 Romani L, Mencacci A, Grohmann U, et al. Neutralizing antibody to interleukin 4 induces systemic protection and Thelper type 1-associated immunity in murine candidiasis. $\mathcal{F}$ Exp Med 1992;176:19-25.
20 Lilic D, Cant AJ, Abinun M, et al. Chronic mucocutaneous candidiasis: I. Altered-antigen stimulated IL-2, IL-4, IL-6 and IFN $\gamma$ production. Clin Exp Immunol 1996;105:205-12.

\section{EGP}

\section{Best Practice articles (formerly "Broadsheets") prepared by the Association of Clinical Pathologists}

\section{Just published}

163 Wilson's disease: acute and presymptomatic laboratory diagnosis and monitoring 2000 D GAFFNEY, GS FELL, DStJ O'REILLY

162 The histological reporting of melanoma 2000 E CALONJE

161 Examination of lung specimens 2000 AR GIBBS, RL ATTANOOS

160 Renal biopsy specimens 2000 PN FURNESS

159 Examination of large intestine resection specimens 2000 SH BURROUGHS, GT WILLIAMS

158 Neuropathology 2000 WR TIMPERLEY

157 Guidelines for the laboratory handling of laryngectomy specimens 2000 TR HELLIWELL

156 Handling oesophageal biopsies and resection specimens and their reporting 2000 NBN IBRAHIM

\section{Recent Publications}

155 Pathological investigation of deaths following surgery, anaesthesia, and medical procedures 1999 RD START, SS CROSS

154 Helicobacter pylori 1999 CAM MCNULTY, JI WYATT (with correction in June issue)

\section{Other Best Practice articles are still available for purchase}

153 The laboratory investigation of vaginal

discharge 1998 KF MACSWEEN, GL RIDGWAY

152 Clinical implications of plasma homocysteine measurement in cardiovascular disease 1998

RA STILL, IFW MCDOWELL

151 Investigation of dyslipidaemias 1997 AF WINDER, W RICHMOND, DT VALLANCE

150 Antenatal serological testing and prevention of haemolytic disease of the newborn 1997 JKM DUGUID

149 Serological diagnosis of gluten sensitive enteropathy 1996 DJ UNSWORTH

148 Laboratory diagnosis of malaria 1996 DC WARHURST, JE WILLIAMS

147 Mycological techniques 1996 KG DAVEY, CK CAMPBELL, DW WARNOCK

146 Macroscopic examination of prostatic specimens 1995 P HARNDEN, MC PARKINSON
Earlier Broadsheets may still be available from the author. A full list can be obtained from the Publications Secretary, Association of Clinical Pathologists, 189 Dyke Road, Hove, East Sussex BN3 1TL.

\section{Prices}

INLAND One copy, $£ 2.50 ; 2-10$ copies (of any one broadsheet or reprint), $£ 2.00$ each; $11-100$ copies (of any one), $£ 1.75$ each; 101 plus copies (of any one), price to be agreed; authors (over 50 free copies), 1.25 each. OVERSEAS One copy, $\$ 6.75 ; 2-10$ copies (of any one broadsheet or reprint), $\$ 5.25 ; 11-100$ copies (of any one), $\$ 3.75 ; 101$ plus copies (of any one), price to be agreed.

Authors $\$ 2.25$. Prices include postage but air mail will be charged extra. Trade discount $10 \%$. All orders (and all changes of address of regular subscribers) should be sent to the Publishing Manager $\bullet$ fournal of Clinical Pathology, BMJ Publishing Group, BMA House, Tavistock Square, London WC1H 9JR. 